\title{
Mathematical Modelling using Gray Markov SCGM(1,1)c of Zambia's Fatal Mining Accidents Between 2001 and 2015
}

\author{
Sunday Mulenga \\ University of Zambia, School of Mines, Department of Mining Engineering \\ sdmulenga13@gmail.com
}

\begin{abstract}
Most mine accidents are caused by human error. The effects of accidents either fatal or not are adverse and range from economical to social. In this paper, the amended Grey Markov model with double exponential smoothing has been used. Predicting fatal accidents will provide the basis of safety assessment and decision making and also help to plan for possible economic and social impacts generated by fatal accidents. The amended Grey Markov combines the advantages of the grey prediction model and the Markov chains and can, therefore, be used on data that is few, has little and stochastic fluctuations. The gray $S C G M(1,1) c$ model is applied to imitate the development tendency of the mine safety accident, and adopt the amended model to improve prediction accuracy, while Markov prediction is used to predict the fluctuation along with the tendency. Finally, the model is applied to forecast the fatal mine accident deaths from 2001 to 2015 in Zambia, and, 2016 fatal mine accidents were predicted. The model predicted the fatal mine accidents results with a relative error of 0.06 and is classified as excellent in the precision test. The proposed model, therefore, possesses a stronger engineering application.
\end{abstract}

Keywords: Grey-Markov, fatal accidents, Prediction 


\subsection{Introduction}

The copper mining industry is one of the largest industries in Zambia. It accounts for about $15 \%$ percent informal employment, 8 percent in gross domestic products (GDP), and 84\% percent in export earnings [1]. However, due to the complexity of the copper mining industry, accidents (fatal and nonfatal) cannot be well controlled and this somehow, restricts the copper production capacity in Zambia. Recent studies have shown that high occupational accidents are found in the mining industry [2-4]. The adversity of these mine accidents can be very bad leading to the suspension of production and poor corporate image for the company or companies involved. Other major consequences of mine accidents include economic and social impacts. The most common economical consequences of mine accidents include the cost to the employer such as lost wages, health costs, and other indirect costs. Indirect costs to the employer include the cost associated with additional hiring and training, time delays due to disruption of the work process and effects, etc. Costs to the family such as reduced income, depletion of reserves and loss of assets; and cost to the community such increased use of social services of the affected family members are also the most common economic effects of mine accidents [5]. Among the mine accidents, fatal accidents are the ones with the most adverse consequences on the employer, family, and the community. Accurately predicting fatal accidents will not only provide the basis of safety assessment and decision making but will also help to plan for possible economic and social impacts generated by fatal accidents.

Copper mining production system is very complex and combined effects on various factors lead to mine accidents. Many scholars and experts consider that human error or people's unsafe behavior is the main reason for mine safety accidents [6-8). Furthermore, studies have shown that human factors are relatively complex and unchangeable [9], therefore, more affecting data is unknown. Mine accident causation by human error meets the criteria of been classified as a grey system. In the recent past, the main methods for predicting safety accidents include experience model, regression model, and the gray prediction method. These models are not suitable to predict mine fatal accidents because most mine accidents are caused by human error. Due to low prediction accuracy as a result of a lack of historical data or inaccuracy and the factor of human error, the Gray Markov SCGM $(1,1)_{\mathrm{c}}$ is the most appropriate tool for predicting fatal mining accidents in Zambia. The Gray Markov combines the advantages of the grey and Markov prediction models. According to the copper mining industry fatal accident statistics provided mine safety department, an amended Gray Markov $\operatorname{SCGM}(1,1) c$ model is proposed. The gray $\operatorname{SCGM}(1,1) c$ model is applied to imitate the development tendency of the mine fatal accidents, and the amended model is used to improve prediction accuracy. The Markov prediction is used to predict the fluctuation along with the tendency to further improve the prediction accuracy on random volatile accident data.

\subsection{Methodology}

2.1.1. Model Selection. According to the actual accident situation in Zambian mines, human error of the miner is not regular; it posses characteristics of being randomly scattered, lack of raw data, and imperfect and uncertain information. Modeling of such data is very difficult using probabilistic statistical and mathematical statistics methods. Gray Markov $\operatorname{SCGM}(1,1) c$ prediction model is the most suitable for model accident information as it possesses characteristics of requiring less information, easy calculation, high accuracy, and so on. The model does not list factors affecting data research objects but finds useful information and explores the inherent laws from their time data sequence establishing a model to predict.

2.1.2. Data Processing. Taking account of the randomness of human error data in mines, original time series $x^{(0)}$ of the mine fatal accident can be expressed:

$$
x^{(0)}=\left\{x^{0}(1), x^{(0)}(2), \cdots, x^{(0)}(n)\right\}
$$

First, $x^{(0)}$ is integrated as follows; 


$$
\bar{x}^{(1)}=\left\{\bar{x}^{(1)}(2), \bar{x}^{(1)}(3), \ldots, \bar{x}^{(1)}(n)\right\}
$$

where;

$$
\bar{x}^{(1)}(k)=\sum_{m=2}^{k} \bar{x}^{(1)}(m), k=2,3, \ldots, n
$$

$\bar{x}^{(0)}$ is a close mean value generated sequence for $\bar{x}^{(0)}$ :

$$
\bar{x}^{(0)}=\left\{\bar{x}^{(0)}(2), \bar{x}^{(0)}(3), \ldots, \bar{x}^{(0)}(n)\right\}
$$

where

$$
\bar{x}^{(1)}(k+1)=\frac{x^{(0)}(k+1)+x^{(0)}(k)}{2}
$$

2.1.3. Response Function. Random human error leads to accidents in Zambian copper mines. Therefore, the majority of accidents are dynamic. Given that an integral sequence of mine fatal accidents time series is expressed as $\left\{\bar{x}^{(0)}(k)\right\}$ that is associated with satisfaction trend of non-homogeneous index discrete function expressed as $f_{r}(k)=b e^{a(k-1)}-c$, thus the data of $\bar{x}^{(1)}(k)$ is fit to $f_{r}(k)$. According to the gray system cloud forecast method, the system gray $\operatorname{SCGM}(1,1)_{c}$ prediction model can be expressed as;

$$
\frac{d \bar{x}^{(1)}(k)}{d k}=a \bar{x}^{(1)}(k)+u, k \geq 2
$$

Its time response function can be expressed as follows;

$$
\bar{x}^{(1)}(k)=\left[\bar{x}^{(1)}(1)+\frac{\mathrm{U}}{\mathrm{a}}\right] \cdot e^{a k}-\frac{U}{a}
$$

Where;

$$
\begin{gathered}
a=\frac{\ln \sum_{k=3}^{n} \bar{x}^{(0)}(k-1) \bar{x}^{(0)}(k)}{\sum_{k=3}^{n}\left(\bar{x}^{(0)}(k-1)\right)^{2}} \\
b=\frac{(n-1) \sum_{k=2}^{n} e^{a(k-1)} \bar{x}^{(1)}(k)-\left(\sum_{k=2}^{n} e^{a(k-1)}\right)\left(\sum_{k=2}^{n} \bar{x}^{(1)}(k)\right)}{(n-1) \sum_{k=2}^{n} e^{2 a(k-1)}-\left(\sum_{k=2}^{n} e^{a(k-1)}\right)^{2}} \\
c=\frac{1}{n-1}\left[\left(\sum_{k=2}^{n} e^{a(k)}\right) b-\sum_{k=2}^{n} \bar{x}^{(1)}(k)\right]
\end{gathered}
$$

Given $\bar{x}^{(1)}(1)=b-c, U=a c, \bar{x}^{(1)}(k)$ is reverted, the system gray $\operatorname{SCGM}(1,1)_{c}$ prediction model of original data will be expressed as;

$$
\hat{x}^{(0)}(k)=\frac{2 b\left(1-e^{-a}\right)}{1+e^{-a}} \cdot e^{a(k-1)}
$$

The Grey precision index $(Y(k))$, residual error $(\varepsilon(k))$ and the relative error $(\Delta k)$ are calculated as follows

$$
\begin{aligned}
& Y(k)=\frac{x^{0}(k)}{\hat{x}^{(0)}(k)} \\
& \varepsilon(k)=\hat{x}^{(0)}(k)-x^{0}(k)
\end{aligned}
$$




$$
\Delta k=\frac{|\varepsilon(k)|}{x^{0}(k)}
$$

Indicators in equations 10,11, and 12 are gray fitting accuracy indicators, which reflect the degree of deviation of the predicted values to the original data set.

2.2. Establishment of the Residual Amended SCGM $(1,1)$ c Model. Statistics data fluctuations of Zambian copper mining fatal accidents are larger, and the regularity is not very strong as the uncertainty of the person's behavior. Therefore, the prediction accuracy should not be too good if the $\operatorname{SCGM}(1,1) c$ model is solely applied to predict accident deaths of Zambian copper mines. To improve the prediction rate and better meet the actual situation, the prediction model should be corrected to improve accuracy. The amended principle and steps are as follows.

(1) The first time residuals data sequence is got following the predicted value and actual value:

$$
\begin{aligned}
\varepsilon^{(0)}(k)=\hat{x}^{(0)}(k)-x^{0}(k), k=1,2,3, \ldots, n \\
\varepsilon^{(0)}(k)=\left\{\varepsilon^{0}(1), \varepsilon^{0}(2), \ldots, \varepsilon^{0}(n)\right\}
\end{aligned}
$$

(2) Processing residuals correction sequence.

Given that $M=\left(1+e^{-a}\right)^{-1}\left(1-e^{-a}\right)^{b}$, the $\operatorname{SCGM}(1,1) c$ prediction will be expressed as $\hat{x}^{(0)}(k)=$ $2 e^{a(k-1)} M$. If $\varepsilon^{(0)}(k) \geq 0,(k=1,2,3, \ldots, n)$ the residual amended $\operatorname{SCGM}(1,1)$ c model will be expressed as $\hat{\varepsilon}^{(0)}(k)=2 e^{a_{1}(k-1)} M_{1} \cdot a_{1,1}$, and $b_{1}$ can be obtained following $a, M$, and $b$ used method. The first time residuals corrected $\operatorname{SCGM}(1,1) c$ model can be expressed as follows:

$$
\hat{x}_{\varepsilon_{1}}{ }^{(0)}(k)=2\left(e^{a(k-1)} M-e^{a_{1}(k-1)} M_{1}\right)
$$

If $\varepsilon^{(0)}(k)<0,(k=1,2,3, \ldots, n)$, the first time residuals corrected $\operatorname{SCGM}(1,1) c$ model can be expressed as follows:

$$
\hat{x}_{\varepsilon_{1}}{ }^{(0)}(k)=2\left(e^{a(k-1)} M+e^{a_{1}(k-1)} M_{1}\right)
$$

The first time the residuals amendment did not meet the forecast accuracy required. Therefore, a second residual correction according to the principals in section 2.2 was carried out the data set until the required accuracy requirements were met. The residual correction generic model can be expressed as follows:

$$
\hat{x}_{\varepsilon_{2}}{ }^{(0)}(k)=\hat{x}_{\varepsilon_{1}}{ }^{(0)}(k)-2 e^{a_{2}(k-1)} M_{2}
$$

Where; $\varepsilon_{1}{ }^{(0)}(k) \geq 0,(k=1,2,3, \ldots, n)$, and;

$$
\hat{x}_{\varepsilon_{2}}{ }^{(0)}(k)=\hat{x}_{\varepsilon_{1}}{ }^{(0)}(k)+2 e^{a_{2}(k-1)} M_{2}
$$

Where; $\varepsilon_{1}{ }^{(0)}(k) \leq 0,(k=1,2,3, \ldots, n)$

\subsection{Establishment of Amended Markov SCGM(1,1)c Model.}

The $\operatorname{SCGM}(1,1)_{c}$ prediction fitting curve is essentially an exponential curve, and the prediction result is a relatively smooth curve. Because human error accidents are the main part of mine accidents, the $\operatorname{SCGM}(1,1)_{c}$ model solely applied cannot meet forecast accuracy requirements. Markov's theory has no aftereffect, that is to say, "the future state of the system is only related to the current state, and has nothing to do with the past state." Meanwhile, the Markov model is adopted to predict state trends through probability transfers, it can adapt to the randomness and variability of state. Applying Markov theory to correct the $\operatorname{SCGM}(1,1)_{\mathrm{c}}$ prediction model of Zambian copper mine fatal accidents can better solve the variability and randomness of accidents caused by human errors to improve the prediction accuracy.

2.3.1. State Divided. The annual change of the number of deaths in mine accidents is a dynamic nonstationary random process, and thus the prediction fitting precision indicators also are variability and randomness. Because boundary and connotation of the different annual states are changeable, an adaptive 
state divided criterion needs to be determined, and the criterion should be consistent with the basic timing trend of the Zambia Copper Mines fatal accidents. Thus, $(k)$ was divided into $m$ states, and each state can be expressed as:

$$
E_{i} \in\left[\otimes_{1 i}, \bigotimes_{2 i}\right], i=1,2, \ldots, m .
$$

Where; $\otimes_{1 i}=Y(k)+A_{i}, \otimes_{2 i}=Y(k)+B_{i}$

In the formula, $E_{i}$ is expressed as $i$ state, $\bigotimes_{1 i}$ and $\bigotimes_{2 i}$ are, respectively, expressed as the upper and lower bounds of the $i$ state, and $A_{i}$ and $B_{i}$ are constants determined according to prediction data. Because $(k)$ is a time function, $\otimes_{1 i}$ and $\otimes_{2 i}$ will be changed with time, so the state possesses variability.

When the state is divided, the numbers of different intervals are reasonably divided according to the actual situation. If raw data are less, the interval division should be less to increase the number of transfers between the various states, and thus the transfer law can be more objectively reflected between states. Conversely, if raw data are more, the interval division should be less to excavate more information from a large number of data to improve the prediction accuracy. It is suitable to adapt the clustering classification method to determine class number and classification intervals due to fewer data and the uncertain status of human error accidents in the copper mines.

2.3.2. Construction of State Transition Rate Matrix. The original number of samples is expressed as $M_{i j}(k)$ from the state $E_{i}$ transiting to the sate $E_{j}$ by $k$ step, and the number of occurrences of the state $E_{i}$ is expressed as $M_{i}$, so state transition probability is expressed as follows:

$$
P_{i j}(k)=\frac{M_{i j}(k)}{M_{i}},, i, j=1,2, \ldots, M .
$$

$\mathrm{M} \times \mathrm{M}$ state transition probability matrix can be obtained as follows;

$$
P(k)=\left[\begin{array}{ccc}
P_{11}(k) & P_{12}(k) \cdots & P_{13}(k) \\
P_{21}(k) & P_{22}(k) & P_{23}(k) \\
\vdots & \vdots & \vdots \\
P_{m 1}(k) & P_{m 2}(k) & P_{m 3}(k)
\end{array}\right]
$$

2.3.3. The Predictive Value Determined. The state transition probability matrix $(k)$ reflects all statistical regularities of state transition, and the future system state steering can be predicted by investigating the matrix. In the actual analysis of the process, one step transition probability matrix (1) is generally only examined. Given that predicted moment object is in the state $E_{k}$, investigating the $k$ row of (1) can get the following.

(1) If $\max \mathrm{P}_{i j}=\mathrm{P}_{k l}$, the next time system should most likely shift from the state $E_{k}$ to the state $E_{l}$.

(2) If there are two or more probability values identical or similar to $k$ row in the matrix $P(1)$, the future state steering will be difficult to determine; it needs to consider probability transition matrix $P(2)$ or $P(n)(n \geq 3)$.

The system's future state will be determined by investigating the state transition probability matrix, and the gray change interval of relative prediction value in the future moments also will be determined; it can be expressed as $\left[\bigotimes_{1 i}, \bigotimes_{2 i}\right]$. The predicted value of the future moment can be expressed as the interval median as $Y^{\prime}(k)$. 


$$
Y^{\prime}(k)=\frac{x^{0}(k)}{\hat{x}^{(0)}(k)}=\frac{1}{2}\left(\bigotimes_{1 i}+\bigotimes_{2 i}\right)=Y(k) \times \frac{1}{2}\left(A_{i}+B_{i}\right)
$$

\subsection{Error Analysis}

To examine the precision of the proposed model, further tests are required to determine the error between the forecast value and actual value. In this study, the relative error (RPE) analysis was adopted to assess the model precision.

\subsubsection{Relative error analysis}

Relative percentage error (RPE) compares the real and forecast values at specific time k. RPE is defined as Equation 12. The total model precision can be defined by average relative percentage error (ARPE) as follows

$$
A R P E=\frac{1}{n} \sum_{k=1}^{n} R P E, \text { where } \mathrm{k}=2,3,4, . .
$$

\subsection{Model Precision Test}

Table 1[10] was used to assess the precision level of the results generated by a model.

Table 1: Classification of prediction results

\begin{tabular}{ccc}
\hline Prediction Precision Grade & P & C \\
\hline Excellent & $>0.95$ & $<0.35$ \\
Good & $>0.8$ & $<0.5$ \\
Fair & $>0.7$ & $<0.55$ \\
Poor & $<0.7$ & $>0.65$ \\
\hline & $C=\frac{S_{2}}{S_{1}}$ & $(24)$
\end{tabular}

Where; $S_{1}^{2}=\frac{1}{n-1} \sum_{k=1}^{n}\left[x^{0}(k)-\bar{x}\right]^{2}, S_{1}^{2}=\frac{1}{n-1} \sum_{k=1}^{n}\left[\varepsilon^{0}(k)-\bar{\varepsilon}\right]^{2}, \quad \bar{\varepsilon}=\frac{1}{n} \sum_{k=1}^{n} \varepsilon^{0}(k), \bar{x}=\frac{1}{n} \sum_{k=1}^{n} x^{0}(k)$,

$$
P=p\left\{\left|\varepsilon_{i}^{0}(i)-\vec{\varepsilon}_{i}^{0}(i)\right|<0.6675 S_{1}\right\}
$$

\subsection{Application}

The original data sequence of mine fatal accidents from 2001 to 2014 in the Zambian Mining industry are shown in Table 1, and then we are applying the Grey-Markov forecasting model to forecast the mine fatal accidents, according to the methodology proposed in section 2 , the forecasting steps are as follows.

\subsection{Building GM $(1,1)$ forecasting model}

Based on the original data of fatal mine accidents from 2001 to 2015 , the value $(a=-0.0408, b=-596.85)$ can be obtained after calculating, then the grey forecasting model can be established; 


$$
\hat{x}^{(0)}(k)=24.36 e^{-0.0408(k-1)}
$$

The forecast value is calculated out by Equation 23, see Table 1

Table 1: Real Value and Forecast Value of Fatal Mine Accidents from 2001 to 2015

\begin{tabular}{rcccc}
\hline Year & Real Value & Forecast Value & Relative Error & $\begin{array}{c}\text { Grey Precision } \\
\text { Index }(\%)\end{array}$ \\
\hline $\mathbf{2 0 0 1}$ & 23 & 24.36 & 0.06 & 94 \\
$\mathbf{2 0 0 2}$ & 17 & 23.39 & 0.38 & 73 \\
$\mathbf{2 0 0 3}$ & 21 & 22.45 & 0.07 & 94 \\
$\mathbf{2 0 0 4}$ & 19 & 21.55 & 0.13 & 88 \\
\hline $\mathbf{2 0 0 5}$ & 34 & 20.69 & 0.39 & 164 \\
\hline $\mathbf{2 0 0 6}$ & 18 & 19.86 & 0.10 & 91 \\
$\mathbf{2 0 0 7}$ & 15 & 19.07 & 0.27 & 115 \\
\hline $\mathbf{2 0 0 8}$ & 21 & 18.31 & 0.13 & 97 \\
\hline $\mathbf{2 0 0 9}$ & 17 & 17.57 & 0.03 & 83 \\
\hline $\mathbf{2 0 1 0}$ & 14 & 16.87 & 0.21 & 123 \\
\hline $\mathbf{2 0 1 1}$ & 20 & 16.20 & 0.19 & 77 \\
$\mathbf{2 0 1 2}$ & 12 & 15.55 & 0.30 & 80 \\
\hline $\mathbf{2 0 1 3}$ & 12 & 14.93 & 0.24 & 91 \\
\hline $\mathbf{2 0 1 4}$ & 13 & 14.33 & 0.10 & 116 \\
\hline
\end{tabular}

\subsection{Building of the Residual Amended SCGM $(1,1)$ c Model}

Based on the relative error between the real and forecasted data between of fatal mine accidents between 2001 to 2014, the residual Amended $\operatorname{SCGM}(1,1)_{C}$ was developed with a value of $\mathrm{a}_{1=-0.1984 \text { and } \mathrm{b}_{1}=-}$ 61.3129. The first time residuals corrected $\operatorname{SCGM}(1,1) c$ model can be expressed as follows:

$$
\hat{x}_{\varepsilon_{1}}{ }^{(0)}(k)=2\left(e^{-0.0408(k-1)} 12.18 \pm e^{-0.2107(k-1)} 6.06\right)
$$

For $\varepsilon_{1}{ }^{(0)}(k) \leq 0,+$ is used and $\varepsilon_{1}{ }^{(0)}(k) \geq 0$, - is used.

The forecast values as calculated out from equation 23 are shown in Table 2.

\begin{tabular}{|c|c|c|c|c|}
\hline Year & Real Value & Forecast Value & Relative Error & $\begin{array}{l}\text { Grey Precision } \\
\text { Index }(\%)\end{array}$ \\
\hline 2001 & 23 & 12 & 0.47 & 187.98 \\
\hline 2002 & 17 & 13 & 0.21 & 126.42 \\
\hline 2003 & 21 & 14 & 0.32 & 146.89 \\
\hline 2004 & 19 & 15 & 0.22 & 127.83 \\
\hline 2005 & 34 & 26 & 0.23 & 129.90 \\
\hline 2006 & 18 & 15 & 0.15 & 117.16 \\
\hline 2007 & 15 & 15 & 0.00 & 97.51 \\
\hline 2008 & 21 & 21 & 0.00 & 98.44 \\
\hline 2009 & 17 & 15 & 0.11 & 112.65 \\
\hline 2010 & 14 & 15 & 0.07 & 94.36 \\
\hline 2011 & 20 & 18 & 0.10 & 111.94 \\
\hline 2012 & 12 & 14 & 0.17 & 84.61 \\
\hline 2013 & 12 & 14 & 0.17 & 86.90 \\
\hline 2014 & 13 & 13 & 0.00 & 96.94 \\
\hline 2015 & 16 & 15 & 0.06 & 110.24 \\
\hline
\end{tabular}

Table 2: Forecast Value of Fatal Mine Accidents from 2000 to 2015 using the residual SCGM $(1,1)_{c}$

3.2. Building of the second Residual Amended SCGM $(1,1)$ c Model 
Based on the relative error between the real and forecasted data between of fatal mine accidents between 2001 to 2015, the residual Amended $\operatorname{SCGM}(1,1)_{C}$ was developed with a value of $\mathrm{a}_{2=-}-0.2107$ and $\mathrm{b}_{2}=-$ 43.273. The first time residuals corrected $\operatorname{SCGM}(1,1) c$ model can be expressed as follows:

$$
\hat{x}_{\varepsilon_{2}}{ }^{(0)}(k)=\hat{x}_{\varepsilon_{1}}{ }^{(0)}(k) \pm 9.08 e^{-0.2107(k-1)}
$$

For $\varepsilon_{2}{ }^{(0)}(k) \leq 0,+$ is used and $\varepsilon_{2}{ }^{(0)}(k) \geq 0$, - is used.

The forecast values as calculated out from equation 14 are shown in Table 3.

\begin{tabular}{|c|c|c|c|c|}
\hline Year & Real Value & Forecast Value & Relative Error & $\begin{array}{c}\text { Grey Precision } \\
\text { Index }(\%)\end{array}$ \\
\hline 2001 & 23 & 21 & 0.083 & 109 \\
\hline 2002 & 17 & 20 & 0.198 & 84 \\
\hline 2003 & 21 & 20 & 0.050 & 105 \\
\hline 2004 & 19 & 20 & 0.044 & 96 \\
\hline 2005 & 34 & 30 & 0.120 & 114 \\
\hline 2006 & 18 & 18 & 0.009 & 99 \\
\hline 2007 & 15 & 13 & 0.146 & 117 \\
\hline 2008 & 21 & 19 & 0.083 & 109 \\
\hline 2009 & 17 & 17 & 0.019 & 102 \\
\hline 2010 & 14 & 14 & 0.026 & 103 \\
\hline 2011 & 20 & 19 & 0.045 & 105 \\
\hline 2012 & 12 & 13 & 0.092 & 92 \\
\hline 2013 & 12 & 13 & 0.106 & 90 \\
\hline 2014 & 13 & 12 & 0.045 & 105 \\
\hline 2015 & 16 & 15 & 0.033 & 103 \\
\hline
\end{tabular}

\subsection{Dividing states for Markov forecasting.}

According to grey precision index (see Table 3) from 2000 to 2015, four transitional states are divided as follows, and the circumstances that the grey precision index lies in the state are listed in Table 5.

$$
\otimes_{1}=[80,90], \bigotimes_{2}=[90,100], \bigotimes_{3}=[100,110], \bigotimes_{4}=[110,120],
$$

The states are described as shown in Table 4.

Table 4: State division of Zambian Mining industry fatal accidents

\begin{tabular}{ccc}
\hline No. of State & Dividend State & Precision Index Range $(\%)$ \\
\hline $\mathbf{1}$ & Strong decreasing year & $80-90$ \\
$\mathbf{2}$ & Poor decreasing year & $90-100$ \\
$\mathbf{3}$ & Poor increasing year & $100-110$ \\
$\mathbf{4}$ & Strong increasing year & $110-120$ \\
\hline
\end{tabular}

\subsection{Calculating transition probability matrix}

The transition probability was calculated using equation 20 and the methods explained in section 2.3.2. Table 5 shows the initial states to which the grey precision indexes belong. 
Table 5: Initial state of years

\begin{tabular}{|c|c|c|}
\hline Year & Grey Precision Index & Initial state \\
\hline 2001 & 109 & \\
\hline & & 3 \\
\hline 2002 & 84 & \\
\hline & & 1 \\
\hline 2003 & 105 & \\
\hline & & 3 \\
\hline 2004 & 96 & \\
\hline & & 2 \\
\hline 2005 & 114 & \\
\hline & & 4 \\
\hline 2006 & 99 & \\
\hline & & 2 \\
\hline 2007 & 117 & \\
\hline & & 4 \\
\hline 2008 & 109 & \\
\hline & & 3 \\
\hline 2009 & 102 & \\
\hline & & 3 \\
\hline 2010 & 103 & \\
\hline & & 3 \\
\hline 2011 & 105 & \\
\hline & & 3 \\
\hline 2012 & 92 & \\
\hline & & 2 \\
\hline 2013 & 90 & \\
\hline & & 1 \\
\hline 2014 & 105 & \\
\hline & & 3 \\
\hline 2015 & 103 & \\
\hline & & 3 \\
\hline
\end{tabular}

The resulting transitional probability matrix is as follows

$$
\begin{gathered}
P(1)=\left[\begin{array}{cccc}
0 & 0 & 1 & 0 \\
0.33 & 0 & 0 & 0.67 \\
0.125 & 0.25 & 0.8 & 0 \\
0 & 0.5 & 0.5 & 0
\end{array}\right] P(2)=\left[\begin{array}{cccc}
0 & 0.5 & 0.5 & 0 \\
0 & 0.33 & 0.67 & 0 \\
0.125 & 0 & 0.375 & 0.125 \\
0 & 0 & 0.5 & 0.5
\end{array}\right] P(3)=\left[\begin{array}{cccc}
0 & 0.67 & 0 & 0.5 \\
0 . & 0 & 0.33 & 0.67 \\
0.125 & 0.375 & 0.25 & 0 \\
0 & 0 & 1 & 0
\end{array}\right] \\
P(4)=\left[\begin{array}{cccc}
0 & 0.5 & 0 & 0 \\
0 & 0 & 0.67 & 0 \\
0.125 & 0.125 & 0.25 & 0.25 \\
0 & 0 & 1 & 0
\end{array}\right]
\end{gathered}
$$

Due to four states are divided, so latest four years data near to prediction time are selected to make state prediction table (Table 2), the transition steps are defined as 1,2,3,4. 
Table 6: State Prediction

\begin{tabular}{|c|c|c|c|c|c|c|}
\hline & Initial State & Transition & State 1 & State 2 & State 3 & State 4 \\
\hline 2015 & 3 & 1 & 0.125 & 0.25 & 0.8 & 0 \\
\hline 2014 & 3 & 2 & 0.125 & 0 & 0.375 & 0.125 \\
\hline 2013 & 1 & 3 & 0 & 0 & 0 & 0.5 \\
\hline 2012 & 2 & 4 & 0 & 0 & 0.67 & 0 \\
\hline Sum & & & 0.25 & 0.25 & 1.845 & 0.125 \\
\hline
\end{tabular}

\subsection{Calculating forecasting values}

The future fatal mine accident death toll can be predicted based on the four-state transition probability matrices. The fatal mine accident value of 2016 has a high probability of being in state 3 . Therefore, the grey precision index of 2016 is likely to be in state 3, [100,110]. The forecast value of 2015 and 2016 calculated by Markov- Grey is as follows;

$$
\begin{gathered}
Y^{\prime}(2015)=Y(2015) \times \frac{1}{2}\left(A_{i}+B_{i}\right) \\
Y^{\prime}(k)=15 \times \frac{1}{2}(1.00+1.10)=15.75 \\
Y^{\prime}(2016)=Y^{\prime}(2015) \times \frac{1}{2}\left(A_{i}+B_{i}\right) \\
=16 \times \frac{1}{2}(1.00+1.10)=17
\end{gathered}
$$

\begin{tabular}{|c|c|c|c|c|c|}
\hline \multirow[t]{2}{*}{ Year } & \multirow{2}{*}{$\begin{array}{l}\text { Actual } \\
\text { Value }\end{array}$} & \multicolumn{2}{|c|}{ Amended $S C G M(1,1) C$ model } & \multicolumn{2}{|c|}{ Amended Markov $\operatorname{SCGM}(1,1) c$ model } \\
\hline & & Predicted Value & Relative Error & Predicted Value & Relative Error \\
\hline 2012 & 12 & 13.1 & 0.092 & 13.76 & 0.128 \\
\hline 2013 & 12 & 13.3 & 0.106 & 13.94 & 0.139 \\
\hline 2014 & 13 & 12.4 & 0.045 & 13.03 & 0.003 \\
\hline 2015 & 16 & 15.5 & 0.033 & 16.25 & 0.015 \\
\hline 2016 & 19 & & & 17 & 0.105 \\
\hline
\end{tabular}

The forecast values from 2012 to 2015 are also calculated by the Grey-Markov model (see Table 7).

Table 7: Forecasting Results

The forecast values obtained by GM $(1,1)$, Grey-Markov, and the real value are shown in Fig.1, it shows that Grey-Markov forecasting model is better for forecasting fire accidents, the precision of Grey-Markov model is much better than GM $(1,1)$ model. 


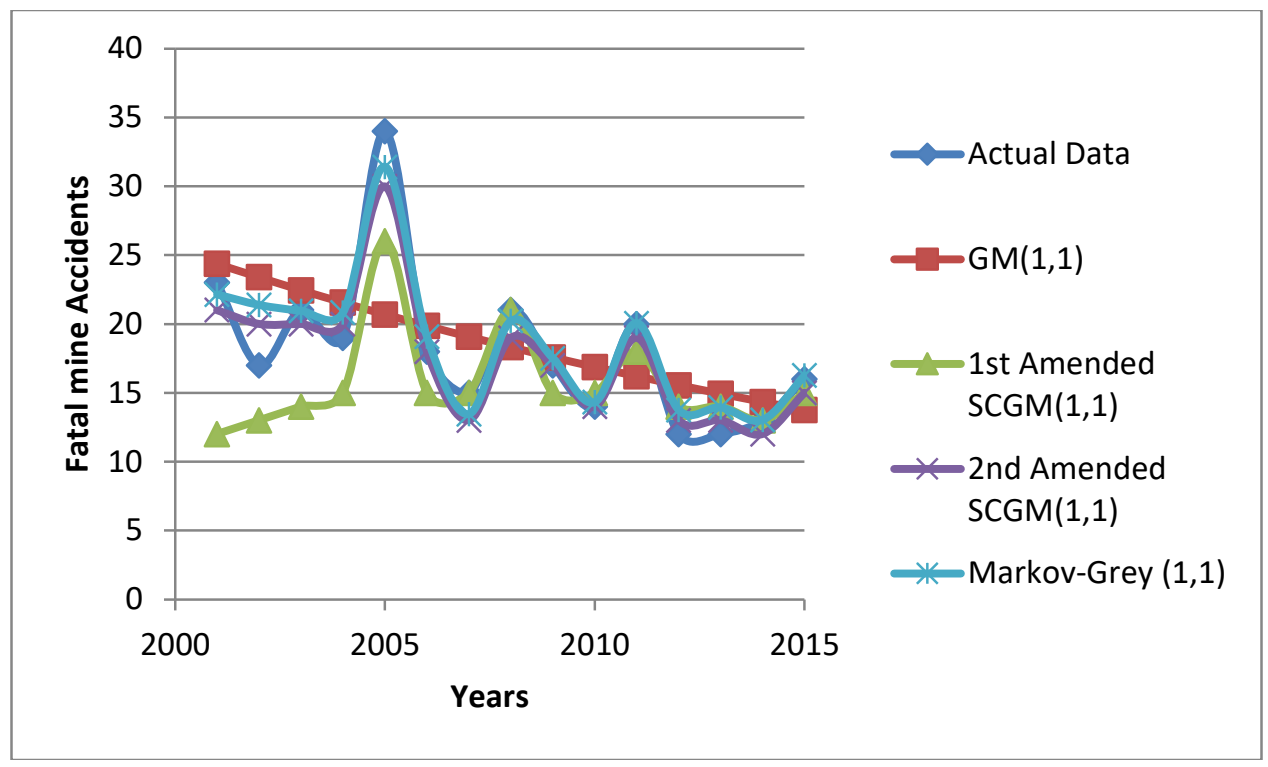

Figure 1: Curve fitting of mine fatal accidents with results from different prediction models

As Figure 1 shows that the fitting degree of the Markov Grey $\operatorname{SCGM}(1,1) c$ model is the best. The second amended $\operatorname{SCGM}(1,1) c$ model is better than the first $\operatorname{SCGM}(1,1) c$ model. the grey model GM $(1,1)$ produced the worst results. The average absolute error for the Markov grey $\operatorname{SCGM}(1,1) \mathrm{c}$ is 0.06 compared to 0.07 for second amended SCGM(1,1)c. The precision test classifies the Markov grey model as excellent i.e $\mathrm{C}=0.046$ which is less than 0.35 and the Value of $\mathrm{P}$ is equal 1 which is greater than 0.95 . the results illustrate that Markov grey $\operatorname{SCGM}(1,1)$ can be used as a good method for fatal accidents in the Zambian mining industry as compared to the other methods. It prediction result correspond to an increasing trend in the next four years.

\subsection{Conclusion}

The Markov grey model is the best prediction accuracy among the four models used for fatal mine accidents in Zambia. It has the lowest relative error and better reflects the relationship between mine fatal accidents and the number of data series. It takes full advantage of historical data and overcomes the data random volatile effects on prediction precision. The proposed model, therefore, possesses strong engineering applications.

\section{References}

[1]. Miller, H.B., Sinkala, T., Renger, R.F., Peacock, E.M., Tabor, J.A., and Burgess, J.L. (2006). Identifying antecedent conditions responsible for the high rate of mining injuries in Zambia. Int $\mathrm{J}$ Occup Environ Health; p329-339.

[2] Prudence, M., Magie, B. and Bante, E. M., (2009). Occupational injuries and fatalities in the copper mining industry in Zambia. Occupational medicine; P119-194

[3] Leigh, J.P., Waehrer, G., Miller, T.R. and Keenan, C. (2004) .Costs of occupational injury and illness across industries. Scand J Work Environ Health; p199-205.

[4]. Kucuker, H. (2006). Occupational fatalities among coal mine workers in Zonguldak, Turkey, 19942003. Occup Med (London); 56:144-146. 
[5]. Camm, T. and Dwyer, G. (2000). Economic consequences of mining injuries. National Institute for Occupational Safety and Health, Spokane Research Laboratory, 315 E. Montgomery Ave., Spokane, WA 99207

[6] Tian, S. C., Li, H. X. and Wang, L. (2006) .Three types of hazard theory and prevention of coal mine accidents. Journal of the China Coal Society, vol. 31, no. 6, pp. 706-710.

[7]. Chen, H., Qi, H., Wang, O. and Long, R. Y. (2007) .The research on the structural equation model of affecting factors of deliberate violation in coalmine fatal accidents in China. System Engineering Theory and Practice, vol. 27, no. 8, pp. 127-136.

[8]. Hong, C., Hui, Q., Xuefeng, S. and Hui, T. (2006) .The structural model of affecting factors of management misconduct in coalmine fatal accidents in China. Journal of the China Coal Society, vol. 31, no. 5, pp. 689-696.

[9]. Patterson, J. M. and Shappell, S. A. (2010) .Operator error and system deficiencies: analysis of 508 mining incidents and accidents from Queensland, Australia using HFACS. Accident Analysis and Prevention, vol. 42, no. 4, pp. 1379-1385.

[10]. Qiao, T. (2016). China's coal mine accident analysis and one million tons mortality prediction. IETI transaction on Computer Volume 2, Issue 1, p61-72 